\title{
Field-Portable, High-Speed GC/TOFMS
}

\author{
Jack A. Syage, Brian J. Nies, Matthew D. Evans, and Karl A. Hanold \\ Syagen Technology, Inc., Tustin, California, USA
}

\begin{abstract}
This work is focused on developing a fast gas chromatograph, time-of-flight mass spectrometer (GC/TOFMS) for man-portable field use. The goal is to achieve a total system solution for meeting performance, size, weight, power, cost, and ruggedness requirements for a laboratory in the field. The core technology will also be adaptable to specific applications including real-time point detection for hazardous chemical releases (e.g., chemical weapons), for biological agent signature identification, and for mobile monitoring platforms (e.g., air, ship, truck). Previously we presented results of a feasibility demonstration for a 30-lb field-portable TOFMS system. In this work we present recent progress in integrating a low-power, high-speed GC and show the capability for accurately recording fast GC transients for targeted compound detection using a quadrupole ion trap, time-of-flight instrument (QitTof). (J Am Soc Mass Spectrom 2001, 12, 648-655) (c) 2001 American Society for Mass Spectrometry
\end{abstract}

$\mathrm{I}$ $\mathrm{n}$ this paper we review our progress toward developing a field-portable, high performance mass spectrometer (MS) system with applications to a variety of fast screening requirements. Previously we reported on a $30-1 b$ class photoionization, quadrupole ion trap, time-of-flight system (PI/QitTof) [1]. More recently we have been developing a low-power, high-speed gas chromatography (GC) sampling system for the QitTof mass analyzer. The intent is to develop a compact system that can perform direct, real-time air and liquid sampling of targeted compounds in complex mixtures in a GC-bypass mode. If a targeted compound is detected during fast screening, the sample can then be subjected to GC/MS analysis for confirmation. This mode of operation achieves high-throughput molecular detection, while affording the option of confirming the results by GC/MS. We present here an overview of our field-portable MS program and then discuss future plans and directions.

The focus of this instrumentation development is to develop a "laboratory-in-the-field" capability to conduct general environmental assessments [2] and a "rapid-response monitor" to quickly and accurately detect toxic compounds, such as chemical agents, resulting from leaks or releases. Other military applications include military force protection and chemical demilitarization. Civilian applications include high-profile event security, emergency first responder protection, and hazardous materials investigations. These applications require systems that can detect in real-time (1-10 s) a host of target compounds at ultralow concentrations (e.g., 1-100 ppb air sampling, 10-100 pg residue) in the presence of a complex matrix that would other-

Published online April 18, 2001

Address reprint requests to Dr. J. A. Syage, Syagen Technology, Inc., 1411 Warner Avenue, Tustin, CA 92780. E-mail: jsyage@syagen.com wise obscure signals of interest due to overlapping signal.

Several manufacturers have developed products for field applications with GC capabilities (e.g., Bruker's MEM line [3], Varian's Saturn Air); however, these are not man-portable. Viking Instruments, recently acquired by Bruker, offers a portable GC/MS (SpectraTrak) billed as a "real-time" monitor for toxic air, water, and soil pollutants; however, its portability is limited by weight and the need to attach a mechanical pump separately [4]. The Hapsite by Inficon is a man-portable GC/MS.

Fieldable MS instruments have been developed for threat detection applications, such as chemical and biological weapons. Oak Ridge National Laboratory has developed a new generation Chemical/Biological MS (CBMS) system based on chemical ionization (CI) and ion trap MS [5]. Field-portable time-of-flight mass spectrometer (TOFMS) systems using electron ionization (EI) have been developed by Kore and Comstock. The Kore instrument is an elegant design that uses an ion pump and membrane introduction system that gives good detection limits, low battery life, and response/ recovery times of a few minutes [6]. Comstock uses a turbomolecular pump that can handle higher sample throughputs; hence direct sampling is feasible with response times on the order of a second. TOFMS apparatus can be made very compact in size. Cotter and coworkers have engineered some miniature TOFMS configurations for the Applied Physics Lab TinyTOF program [7]. These reductions in size are made possible by the availability of compact pumps and fast transient digitizers. TinyTOF is focused on biological detection using laser desorption/ionization. Though novel miniature TOF devices have been demonstrated, the electronics and data acquisition systems have yet to be fully 
miniaturized. Miniature mass spectrometer concepts including quadrupoles, quadrupole arrays [8,9], and magnetic sectors [10] are being developed for spacebased and other applications. These interesting devices may be suitable for field work if interfaced to an appropriate vacuum system.

The characteristics of the present system that differentiate it from existing technologies are: the use of a photoionization source [11], high sampling rate, MS/MS TOF capability, differential pumping for high gas throughput at modest pump capacity, and a system approach that minimizes total weight and power. In this paper we review our previous feasibility work on field-portable TOFMS [1], describe latest results incorporating a low-power, high-speed GC sampler, and present plans for future work leading to a field-deployable instrument.

\section{Experimental}

\section{Photoionization Source}

In this work a photoionization source was used to ionize the eluent of the GC column. This source also offers the potential to conduct rapid screens of targeted compounds in complex mixtures without requiring the gas chromatography (GC) separation stage. This combined utility can then be used to conduct large numbers of analyses and perform on-demand GC/MS for quantitative analysis only when a targeted compound is detected during screening mode. We are currently designing an EI source that can be used to record conventional GC/MS spectra for the purpose of using established library confirmation methods.

Figure 1 illustrates the principle of PI MS. The Syagen PI source imparts an energy that is higher than the ionization potentials (IPs) of typical target molecules, but lower than the IPs of virtually all constituents of air as well as most common solvents. Because these potential interferents are not ionized, the full dynamic range of the mass spectrometer is available for measuring molecules at very low concentrations. Furthermore, because molecules of interest are ionized near their IP thresholds, there is minimal fragmentation to clutter a mass spectrum. These performance features provide tremendous benefits for analyzing mixtures and samples in complex matrices. The method of EI, by contrast, imparts energy that greatly exceeds the dissociation thresholds leading to extensive fragmentation. Photons are generated in a gas discharge cell which, depending on the gas fill, can range in energy from about 8 to 12 $\mathrm{eV}$. Each gas mixture provides non-tunable narrow emission lines.

\section{QitTof Mass Analyzer}

A quadrupole ion trap (QIT), TOFMS was designed for field-portable use. The principal purpose of the QIT is to accumulate ions that are continuously generated

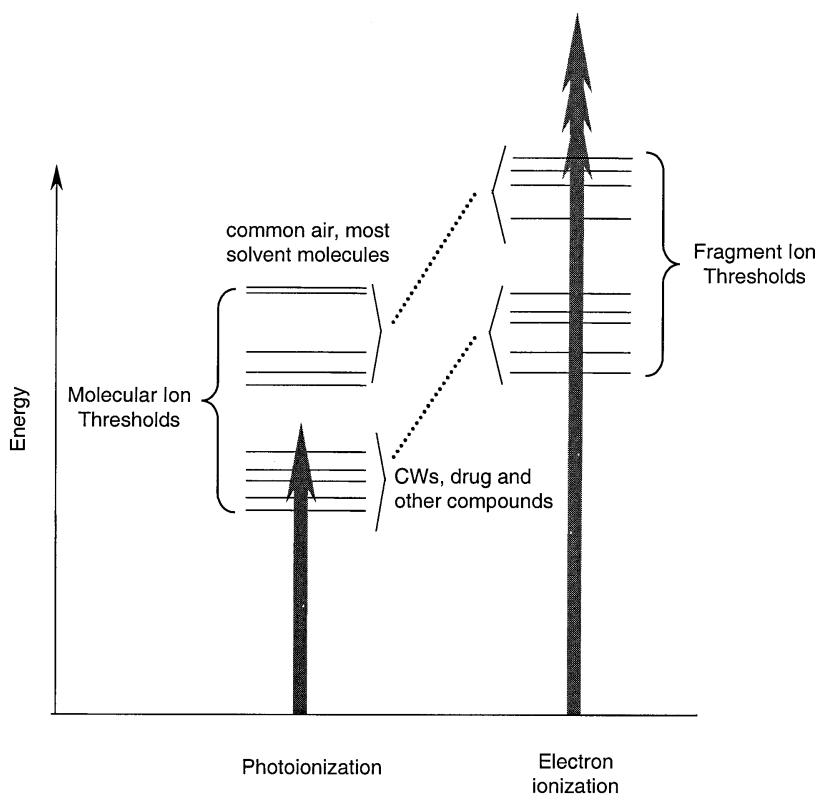

Figure 1. Diagram showing the principle of photoionization for achieving threshold ionization of molecules. The process of electron ionization is shown for comparison.

from the PI source. These ions are then injected into the TOFMS at pulse repetition rates ranging typically from 20-100 Hz. Initial tests of the field portable system used a 22-cm flight-path linear TOF (L-TOF). A machine assembly drawing is given in Figure 2. The details of this prototype system, including weight and power specifications for each component, were described before [1]. In another compact mass spectrometer system a 38-cm long coaxial reflectron TOF (Re-TOF) was tested, as shown in Figure 3. The mass resolutions measured in our laboratory range from $\mathrm{m} / \Delta \mathrm{m}$ values of about 100-200 for the L-TOF to 200-400 for the coaxial Re-TOF configuration at $m / z 181$. Resolutions up to 4000 have been achieved in our $1.2 \mathrm{~m}$ total flight path benchtop Re-TOF (off-axis) using bipolar pulse voltages of up to $1400 \mathrm{~V}$ (the other systems operated with a single extraction pulse of -100 to $-400 \mathrm{~V}$ on the QIT exit endcap). Figure 4 compares the mass resolution for the $\mathrm{m} / \mathrm{z} 139$ molecular ion signal for m-nitrophenol headspace. We plan to employ bipolar pulsing and increase the pulse voltages on the portable systems to improve mass resolution.

The rationale for employing a QitTof mass analyzer compared to a conventional mass-selective instability scanning ion trap mass spectrometer is derived from the following theoretical advantages: (1) fast mass analysis permits higher repetition rates and lower noise per detected ion mass bin, (2) mass resolution is much less sensitive to space-charge repulsion accounting for a greater ion trap capacity, (3) less ion fragmentation in the trap and more accurate intensity measurements due to use of a fixed, low-amplitude RF, and (4) better ion mass accuracy. 


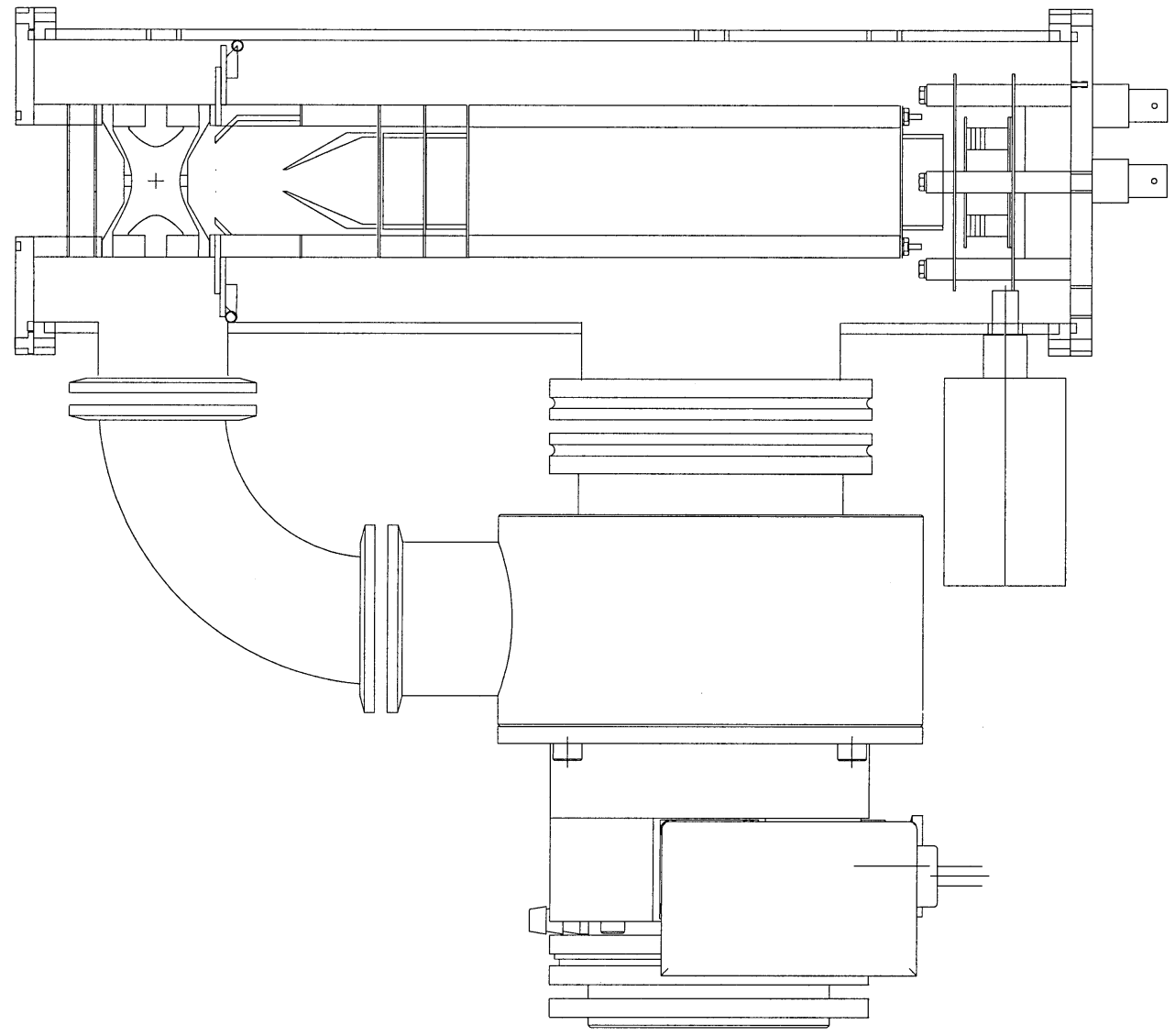

Figure 2. Machine assembly drawing of field-portable prototype used to test feasibility of a 30-lb total detection system. (Ionizer is not shown.) The instrument chamber length is 12 in.

\section{0-lb MS Field-Portable Feasibility}

In a previous publication we reported the feasibility demonstration for a 30-lb PI/QitTof MS system [1]. The PI/QitTof analyzer, consisting of a 22-cm linear flightpath TOF, is mounted on a single flange for quick removal from the vacuum system (Figure 2). The microchannel plate is mounted on a separate flange. The vacuum system employs a split-flow turbomolecular pump (TMP) that provides pumping speeds of about 16 $1 / \mathrm{s}$ in the ion source region and $40 \mathrm{l} / \mathrm{s}$ in the TOF region. The TMP is backed by a $10 \mathrm{l} / \mathrm{min}$ (nominal speed) diaphragm pump that achieves a base pressure of about 1 torr. Under load, the diaphragm pump operated at about $2 \mathrm{l} / \mathrm{min}$ pumping speed at 2 torr, corresponding to a vacuum throughput of 4 torr $1 / \mathrm{min}$ or $5 \mathrm{~atm} \mathrm{~cm}^{3} / \mathrm{min}$. The typical pressures measured in the QIT and TOF regions were 4 mtorr and $5 \times 10^{-5}$ torr, respectively. This corresponds to a differential split ratio of about 25 for pump-out of the QIT region versus throughput into the TOF region. These pressures are somewhat higher than desired. The next generation instrument will attempt to improve pumping speed by

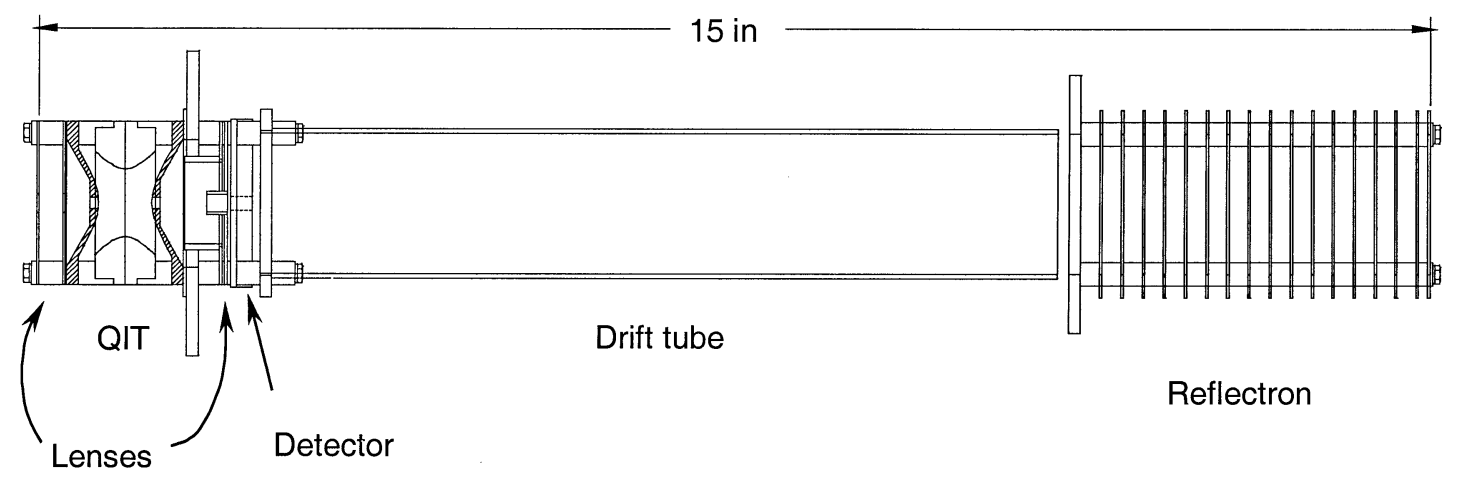

Figure 3. Drawing of a compact coaxial reflectron TOF adaptation of the QitTof mass analyzer. 


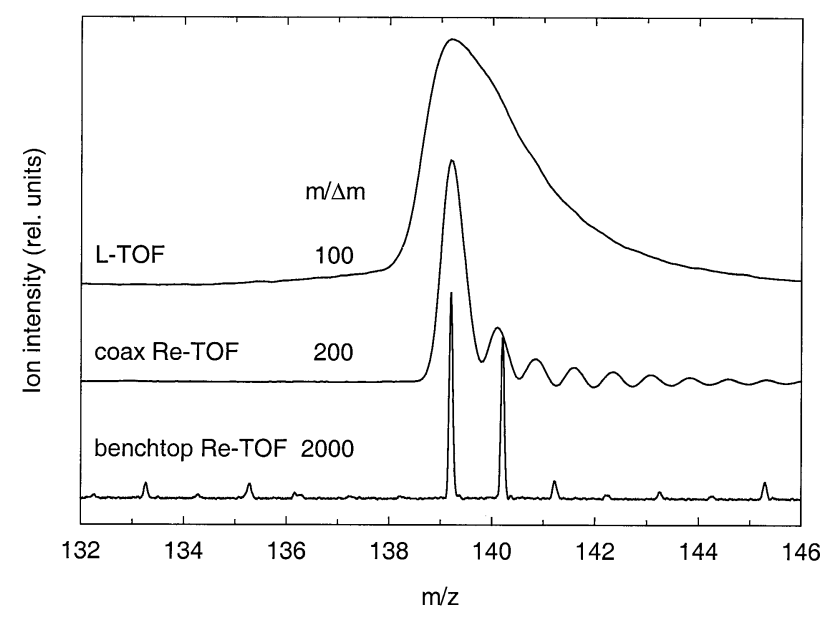

Figure 4. Comparison of mass resolution for compact L-TOF with laptop digitizer, compact coaxial Re-Tof, and benchtop Re-TOF. The coaxial Re-Tof signal can ring if not properly assembled and impedance matched. The bottom spectrum shows a high level of protonated ion at $\mathrm{m} / \mathrm{z} 140$.

better coupling of the turbomolecular pump to the chamber and by reducing the flow rate to 2-3 atm $\mathrm{cm}^{3} / \mathrm{min}(2-3 \mathrm{~mL} / \mathrm{min}$ for GC sampling).

The initial field-portable feasibility tests used a 20 Megasamples/s (MS/s) PCMCIA (Personal Computer Memory Card International Association) digitizer card (National Instruments, Austin, TX) in a laptop computer. This temporary solution was adequate to prove the feasibility of a compact TOFMS system. By operating at low TOF drift tube voltage $(500 \mathrm{~V})$ it was possible to achieve usable mass resolution as evidenced by the mass spectrum of a ppm-level sample of dimethyl methylphosphonate (DMMP) and diisopropyl methylphosphonate (DIMP) in room air in Figure 5. Full MS download and scan rates of up to $200 \mathrm{~Hz}$ were achieved, although this was largely due to the low number of total points recorded per spectrum. In the next generation instrument we plan to install a PCI (Preferred Component Interconnect)-based $500 \mathrm{MS} / \mathrm{s}$

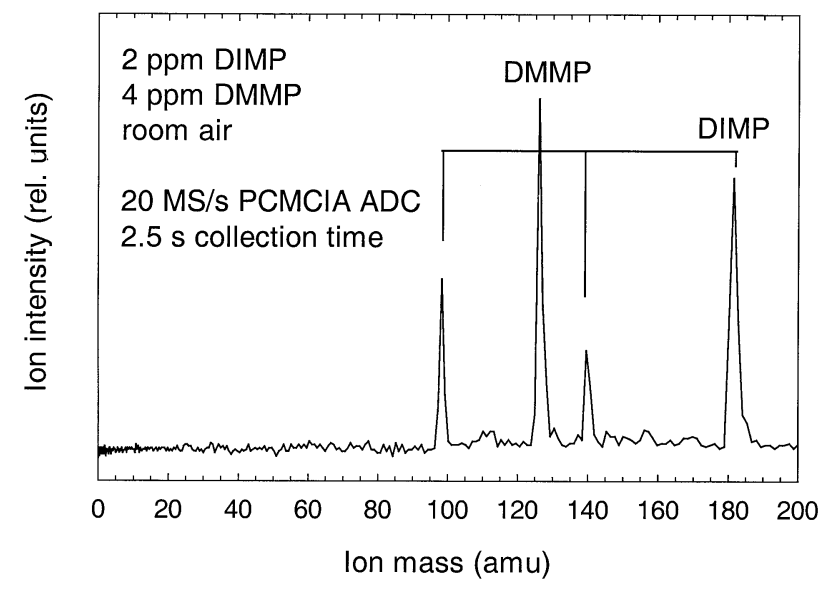

Figure 5. PI/QitTof mass spectra recorded using a $20 \mathrm{MS} / \mathrm{s}$ PCMCIA digitizer card in a laptop computer.

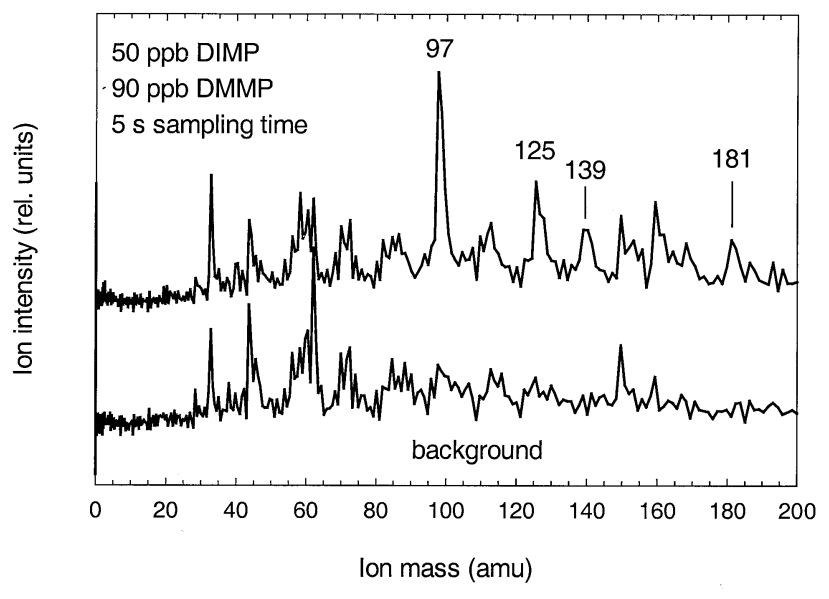

Figure 6. PI/QitTof mass spectra for the 30-lb feasibility instrument illustrating detectability of low concentrations of phosphonates in room air.

digitizer board mounted in an embedded Pentiumbased PC.

A $5 \mathrm{~W}$ high voltage source provided the voltages for the TOF and detector through a resistive voltage divider chain. The next generation instrument will use computer controlled DC/DC converters, while meeting the requirement for a maximum of $120 \mathrm{~W}$ power consumption for the MS system (excluding GC). The PI source power supply consumes about $3 \mathrm{~W}$ and the heaters about $10 \mathrm{~W}$. The major power components are the QIT radio frequency (RF) source and clamping circuit that is activated during pulse out and the pulsing circuits for ion extraction into the TOF. The RF source operates at $1 \mathrm{MHz}$ and has variable amplitude up to about $1200 \mathrm{~V}_{\mathrm{p}-\mathrm{p}}$. This value is capable of stabilizing ion masses up to about $800 \mathrm{amu}$, although only low molecular weight compounds were tested for this project. The extraction pulse circuit incorporates bipolar pulsing, in which opposite polarity pulses are applied to each QIT endcap. The amplitude for each polarity is independently adjustable to $500 \mathrm{~V}$. On average the QIT and pulse extraction circuits consume less than $20 \mathrm{~W}$ of power at $60 \mathrm{~Hz}$ line trigger operation. These are the current electronics specifications. Some of the early feasibility results shown here were obtained using prototype electronic circuits (e.g., an extraction pulse of $\leq 200 \mathrm{~V}$ was applied to the exit QIT endcap).

\section{Direct Air and Liquid Sampling}

A variety of methods were used for direct air and liquid sampling. For direct air sampling either a leak valve or a capillary restrictor was used to throttle the flow of air into the low-pressure PI source. The internal volume of the leak valve inlet was about $0.25 \mathrm{~cm}^{3}$, which accounted for a typical residence time of about $4 \mathrm{~s}$. This inlet was fairly crude, but worked adequately for the relatively volatile compounds reported here (Figures 5-6). In latter work (not shown here) the inlet was 


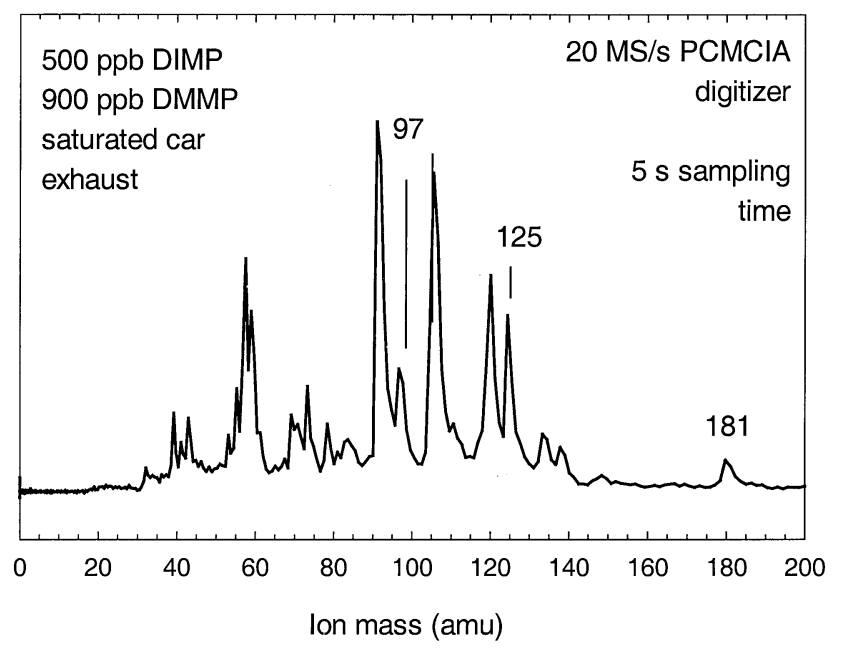

Figure 7. PI/QitTof mass spectra of phosphonate sample in saturated car exhaust.

redesigned with lower internal volume (about $<0.1$ $\mathrm{cm}^{3}$ ), inert surfaces (coated with Silcosteel Restek Corporation, Bellefonte, PA) and higher temperature (up to $300{ }^{\circ} \mathrm{C}$ ) and achieved response time of less than $1 \mathrm{~s}$. The inlet system was also capable of accepting liquid injections. Liquid was injected directly into the heated 1/16-inch ID inlet tube normally used to sample air.

\section{Gas Chromatograph}

The low power GC assembly (RVM Scientific, Inc., Santa Barbara, CA) consumes an average of $15 \mathrm{~W}$ per GC run for temperature programmed ramps and less for isothermal heating. The assembly consists of three heated zones, one for the column and one each for the entrance and exit lines of the GC. The column zone is a toroid of heater and thermocouple wires coiled in parallel with a 10-m long 0.18-mm ID DB1 capillary column (J\&W Scientific, Inc., Folsom, CA), while the entrance and exit zones are constructed of heated 1/16-inch metal sleeves and thermocouple wire. A compact electronics board provides separate heater controls for the GC assembly and the entrance and exit transfer lines. Temperature ramping is controlled using $0-5 \mathrm{~V}$ analog inputs, which operate on all three heated zones in parallel to maintain uniform heating.

The feasibility measurements shown here used isothermal heating of the column. Although temperature ramping can be conducted at up to $20^{\circ} \mathrm{C} / \mathrm{s}$, we decided to keep most variables including temperature at a constant value throughout the initial evaluation of the GC. We did however conduct several different isothermal GC runs at temperatures that varied from 30$100{ }^{\circ} \mathrm{C}$. All of the runs employed syringe injection of a liquid sample of revised California petroleum volatile organic compounds (PVOCs); $1000 \mu \mathrm{g} / \mathrm{mL}$ of benzene, toluene, ethyl-benzene (MTBE), and $\mathrm{o}, \mathrm{m}$, p-xylenes in methanol (Restek Corp., Bellefonte, PA). A 0.5 or $1 \mu \mathrm{L}$ syringe was used to inject about $0.1 \mu \mathrm{L}$ of sample solution with a $0.05 \mu \mathrm{L}$ air gap in less than $1 \mathrm{~s}$ with minimal needle residence time. This fast injection technique proved to be superior to a slow injection with long needle residence time. This resulted in overall good sensitivity and reasonable peak definition and resolution throughout the entire GC run. The liquid injections for this work were performed in a traditional GC injector (SGE GOWMACGM2, from SGE Inc., Austin, TX). The SGE injector has an independently heated injection volume of $130 \mu \mathrm{L}$ that is continuously purged by splitless co-flow of carrier gas. The injector was heated to $150^{\circ} \mathrm{C}$ and ultra pure helium (99.999\%) was introduced as a carrier gas at a relatively high head pressure of $27 \mathrm{psig}$. The exit flow rate from the column at this head pressure was approximately $2 \mathrm{~mL} / \mathrm{min}$. This combination of temperature and pressure was used to produce fast chromatography (elution time less than $100 \mathrm{~s}$ for volatiles) with reasonable resolution while providing the mass spectrometer with an adequate operating pressure regime. However, to test for field applicability without reliance on consumable gases, further evaluation with air as a carrier gas will be necessary.

The detector for all of the GC runs presented here was our $1.2 \mathrm{~m}$ benchtop single reflectron QitTof fitted with the previously described low-pressure photoionization source. This instrument served as the testbed for optimizing the GC. The GC is designed for fieldportable conditions and will be integrated with the next generation of the field-portable QitTof system described below. All spectra were recorded and processed at 60 spectra/s.

\section{Results and Discussion}

\section{QitTof MS Performance}

As a measure of the generality of the PI source for monitoring a broad range of compounds, we tested the PI MS for a large number of chemical agents and chemical weapons convention (CWC) treaty relevant compounds. Table 1 lists all compounds that have been efficiently detected. A 48-compound CWC-relevant compound library was tested, and strong molecular ion signal was obtained for 45 compounds ( $94 \%$ detection rate). Two misses, phosgene and chloropicrin, were not unexpected based on their high ionization potentials. A higher PI energy (e.g., $11.7 \mathrm{eV}$ ) may ionize these compounds; however, this was not verified. The one unexpected miss was benzilic acid, which loses an $-\mathrm{OH}$ group to give a strong signal at $[\mathrm{M}-17]^{+}$. This compound is therefore detectable, but not as a molecular ion. Efficient sample delivery and detection was observed for phosphonic acids (including methylphosphonic and ethylmethylphosphonic acid) as well as alkylethanolamines and alkyl phosphites that are normally difficult to deliver and detect by other MS methods.

Detection limits have been measured on a transport- 
Table 1. Library of CWC-relevant compounds tested using Syagen's PI MS detector

\begin{tabular}{lll}
\hline Schedule-1 compounds & \multicolumn{1}{c}{ Schedule-2 compounds } & Schedule-3 compounds \\
\hline \hline Toxic & Toxic & Precursors \\
VX & BZ & Phosphorus oxychloride \\
GA (Tabun) & Precursors & Dimethyl phosphite \\
GB (Sarin) & Disopropyl methylphosphonate & Trimethylphosphite \\
GD (Soman) & Diethyl methylphosphonate & Diethyl phosphite \\
GF & Dimethyl methylphosphonate & Triethyl phosphite \\
& Diethyl ethylphosphonate & Triethanolamine \\
HD (sulfur mustard) & Diethyl methylphosphonothioate & N-ethyldiethanolamine \\
HN-1 (Nitrogen mustard) & Isopropyl methylphosphonic acid & N-methyldiethanolamine \\
HN-3 & Cyclohexylmethylphosphonic acid & \\
CR & Pinacolyl methylphosphonic acid & \\
CS & Methyl phosphonyl dichloride & Decomposition Products \\
& 3-quinuclidinol & \\
Precursors (Binary) & Benzilic acid & Methylphosphonic acid \\
QL & Pinacolyl alcohol & Ethylmethylphosphonic acid \\
DF & Thiodiglycol & EMPTA \\
& N,N-diethylethanolamine & 2-chlorodiethylsulfide \\
& 1,4-dithiane & Other relevant compounds \\
& Thiodiglycol sulfoxide & VX disulfide \\
& Methylamine & YL \\
& Isopropylamine & \\
\hline
\end{tabular}

able benchtop PI/QitTof system including real agent measured at the Edgewood Chemical and Biological Center Surety Lab at Edgewood, MD. The limits of detection ranged from about 10-100 ppb (2-20 pg consumed) for $5 \mathrm{~s}$ sampling times (based on the linear extrapolation of signal to the $3 \sigma$ background variance). Other classes of compounds, such as drugs and aromatics, give similar detection limits. The 30-lb feasibility study field-portable system generally gave about a factor of 5 poorer detection limits (CW agents weren't tested). About half of this performance compromise can be attributed to the low $20 \mathrm{MS} / \mathrm{s}$ digitizing speed of the field-portable system, which will be upgraded in the next generation instrument. The other half is attributable to the lower pumping speed and reduced sample throughput into the detector.

An air sample containing $50 \mathrm{ppb}$ DIMP and $90 \mathrm{ppb}$ DMMP in room air was made in a 5 gal plastic jug. The resulting spectrum is given in Figure 6. The detection limits for DMMP and DIMP, respectively, were measured for a $5 \mathrm{~s}$ sampling time and gave values of 28 and $25 \mathrm{ppb}$. The DIMP detection limit was based on the stronger 97 amu peak; that of the molecular ion was about a factor of two poorer.

GC-like samples, or other liquid solutions, were analyzed by syringe injection into the air/liquid sampling system. Samples of DIMP and DMMP in methanol were prepared (about $10 \mathrm{ppm}$ by volume, corresponding to about 10 and $12(\mu \mathrm{g} / \mathrm{mL}$, respectively) and sample volumes of $0.1 \mu \mathrm{l}$ were injected. $\mathrm{S} / \mathrm{N}$ ratios of 30 and 80 , respectively, leading to solution detection limits of about $1000 \mathrm{ng} / \mathrm{mL}$ and $450 \mathrm{ng} / \mathrm{mL}$, respectively, were observed.
Specificity was tested by detecting target compounds in a dirty air sample and the ability to distinctly resolve a multitude of compounds in a complex mixture. Figure 7 presents the PI mass spectrum of a mixture consisting of $500 \mathrm{ppb}$ DIMP and $900 \mathrm{ppb}$ DMMP spiked into an air sample saturated with automobile exhaust. The exhaust was collected from the tailpipe of a car in a 5 gal plastic bottle. The bottle was visibly opaque and saturated with water vapor when the spectrum was recorded. The phosphonates were subsequently added by syringe. The DIMP signature peaks 97 amu and 181 amu and the DMMP parent peak at $125 \mathrm{amu}$ are clearly visible for a 5 s sampling time.

The minimal fragmentation and uncomplicated spectra typically observed for molecules enables real-time analysis of multicomponent mixtures. These results show the capability to distinctly detect target compounds in complex matrices while providing rapid screening.

The dynamic range of the PI/QitTof instrument was measured on a benchtop system using the same PI/ QitTof assembly shown in Figure 2. A 4-decade dynamic range and over 3-decade linear range was measured for a single set of instrument conditions [1]. It is expected that this range would decrease by about one decade for the field-portable system due to the poorer detection limits relative to the benchtop version.

\section{GC Interface}

We report preliminary results on the integration of a low power GC to a QitTof MS. As noted in the Experimental section, these experiments were conducted on a 


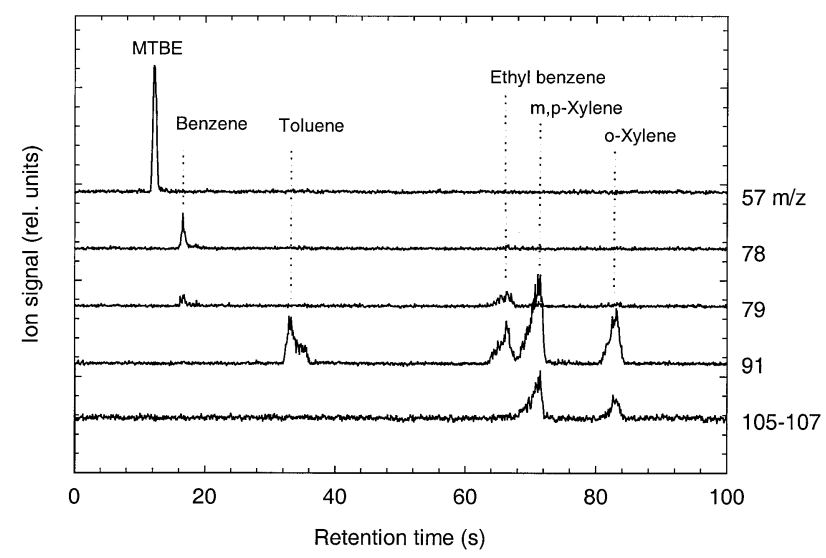

Figure 8. Mass-selected GC traces of Petroleum VOCs (1 mg/ $\mathrm{mL}$ ) sample.

benchtop QitTof instrument to test the feasibility and optimize conditions. The conditions, however, are compatible with the field-portable instrument and future work will integrate the GC device to the next generation field-portable instrument now under development. The goal for this paper was to demonstrate fast chromatography (less than two minutes run time) with reasonable separation and response for a mixture of analytes in a common matrix.

The range of experimental conditions was described in the Experimental section. Figures 8 and 9 show results of a $0.1 \mu \mathrm{L}$ injection of the PVOC sample for isothermal heating at $40{ }^{\circ} \mathrm{C}$ and column head pressure of 27 psig He. The mass-selected GC traces in Figure 8 show characteristic masses for all of the compounds except for MTBE. By direct liquid capillary infusion we observed a dominant $\mathrm{m} / \mathrm{z} 73$ peak corresponding to loss of $\mathrm{CH}_{3}$ (from the tertiary carbon). By GC injection, the dominant peak is at $m / z 57$, which corresponds to loss of the $\mathrm{OCH}_{3}$ group. Assuming these are fragments from

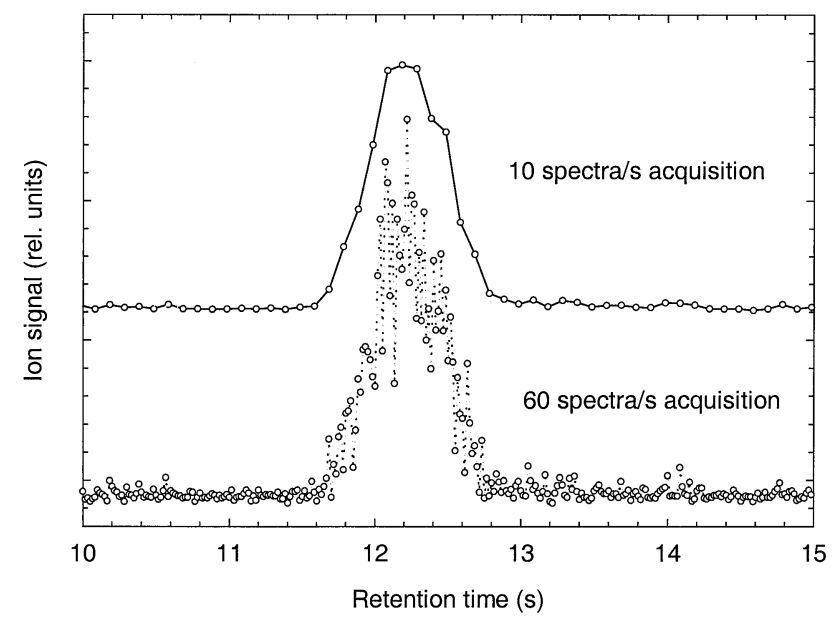

Figure 9. Comparison of $\mathrm{m} / \mathrm{z} 57$ trace recorded for two different acquisition rates. In both cases, mass spectra were recorded and stored at $60 \mathrm{spectra} / \mathrm{s}$. The top spectrum corresponds to summing every 6 spectra. molecular ion dissociation, it is not clear why they should differ depending on sampling method. The $57 / 73$ ratio did not depend significantly on He pressure or GC and PI temperature over the modest ranges tested.

All sample constituents are clearly resolved in less than $90 \mathrm{~s}$ except for $\mathrm{m}$ - and $\mathrm{p}$-xylenes. The early eluting analytes MTBE and benzene have superior peak shape and resolution (less than $1 \mathrm{~s}$ ) while the late eluting compounds demonstrate fronting problems and moderate resolution. The inclusion of temperature ramping to the column heating method and a larger bore column to reduce the head pressure should alleviate these problems. Figure 9 compares the $\mathrm{m} / \mathrm{z} 57$ GC-peak of MTBE recorded at 60 spectra and 10 spectra/s. In both cases, MS acquisition was $60 \mathrm{~Hz}$; however, in the latter case, every 6 spectra were summed. These results show the capability for QitTof to fully resolve the fast transients that result from high-speed GC.

The rated average power consumption of the GC is $15 \mathrm{~W}$ for a temperature ramp from ambient to $240^{\circ} \mathrm{C}$. For the low temperature isothermal heating used in this work we estimate a power consumption of $<10 \mathrm{~W}$. These low power ratings are important for field portable applications, particularly when using battery power. We have not tried air as a carrier gas yet, but based on the Van Deemter equations and observed performance for semivolatiles on similar columns, we believe that only minor degradation of performance will result [12].

\section{Future Plans}

The purpose of the field-portable high-speed GC/ TOFMS is to provide a capability to perform rapid screening of targeted compounds in air or liquids. This can then be followed by confirmatory analysis using GC/MS if a positive signal is registered for a targeted compound. TOFMS enables rapid screening for essentially an unlimited number of ion masses. The next generation instrument under development will employ a dual PI/EI ionizer, the latter for recording conventional GC/MS spectra for library matching to EI-MS spectra.

The field-portable rapid screening MS can accommodate a variety of front-end sampling devices in addition to GC. We are examining a preconcentrator with about a $30 \mathrm{~s}$ cycle time to greatly improve the detection limits for direct air sampling. We are also working toward implementing a pyrolyzer for collecting biological particles, such as bacteria and spores, and thermally decomposing them into molecular signatures for making positive identification of threats such as biological weapons and other pathogens.

\section{Conclusions}

We have described our progress in developing a highspeed GC/TOFMS for man-portable field use. This 
work builds on our previous feasibility demonstration of a 30-lb field-portable TOFMS system. The features that distinguish the current work from previous work include:

- Novel threshold photoionization source

- QitTof mass analyzer

- High-speed TOF; up to $200 \mathrm{~Hz}$ full mass spectra recording rate

- Integration with low-power, high-speed GC

- 30-40 lb feasibility

We anticipate a demand for a GC/MS that can operate in two modes: rapid screening (10 s) of complex mixtures using PI MS, and on-demand GC/MS using EI and library matching for detailed analysis. This strategy would provide maximum efficiency and sampling rates, particularly if operating on battery power.

\section{Acknowledgments}

We wish to acknowledge Dr. Mark Hanning-Lee (Syagen) for his software contributions. We are grateful for the support of this work by the Soldier, Chemical and Biological Command (SBCCOM) of the U. S. Army and by the Defense Threat Reduction Agency (DTRA).

\section{References}

1. Syage, J. A.; Hanning-Lee, M. A.; Hanold, K. A. Field Anal. Chem. Technol. 2000, 4, 204.

2. McDonald, W.; Erickson, M.; Abraham, B.; Robbat, A. Env. Sci. Technol. 1994, 28, 336A.

3. Baykut, G.; Franzen, J. TRAC 1994, 13, 267.

4. Eckenrode, B.A. Field Anal. Chem. Technol. 1998, 2, 3.

5. Hart, K. J.; Wise, M. B.; Griest, W. H.; Lammert, S. A. Field Anal. Chem. Tech. 2000, 4, 93.

6. White, A. J.; Blamire, M. G.; Corlett, C. A.; Griffiths, B. W.; Martin, D. M.; Spencer, S. B.; Mullock, S. J. Rev. Sci. Instrum. 1998, 69, 565.

7. Cornish, T. J.; Cotter, R. J. Anal. Chem. 1997, 69, 4615.

8. Orient, O. J.; Chutjian, A.; Garkanian, V. Rev. Sci. Instrum. 1997, 68, 1393.

9. Ferran, R. J.; Boumsellek, PS. J. Vac. Sci. Technol. 1996, 14, 1258.

10. Sinha, M. P.; Tomassian, A. D. Rev. Sci. Instrum. 1991, 62, 2618.

11. Syage, J. A.; Evans, M. D.; Hanold, K. A. Photoionization Mass Spectrometry, Amer. Lab. 2000, 32, 24.

12. Grob, R. L., The Modern Practice of Gas Chromatography, 3rd ed; Wiley Interscience, New York, 1995. 